\title{
Sistemas de explotação de seringueira utilizados em clones asiáticos Prang Besar no Oeste paulista
}

\author{
Juliano Quarteroli Silva ${ }^{(1)}$, Mario Ivo Tavares de Souza ${ }^{(2)}$, Paulo de Souza Gonçalves ${ }^{(1)}$ e Raquel Nakazato Pinotti(3) \\ (1)Instituto Agronômico, Programa Seringueira, Caixa Postal 28, CEP 13001-970 Campinas, SP. E-mail: quarteroli@gmail.com, \\ paulog@iac.sp.gov.br (2)Fazenda Santa Gilda, Grupo Rodobens Agrícola e Pecuária Ltda., Rod. Marechal Rondon, Km 565, CEP 16700-000 \\ Guararapes, SP. E-mail: marioivo@uol.com.br ${ }^{(3)}$ Apta Regional Centro-Oeste/UPD-Bauru, Av. Rodrigues Alves, no 40, CEP 17030-000 Bauru, SP. \\ E-mail: raquelnakazato@aptaregional.sp.gov.br
}

\begin{abstract}
Resumo - O objetivo deste trabalho foi avaliar o desempenho produtivo e os aspectos físiológicos e econômicos de quatro clones asiáticos Prang Besar de seringueira [Hevea brasiliensis (Willd. ex A. Juss.) Muell. Arg.], sob diferentes sistemas de sangria. O experimento foi instalado na Fazenda Santa Gilda, Município de Guararapes, SP, em delineamento de blocos ao acaso, com parcelas subdivididas no tempo. Os tratamentos principais foram os clones PB 260, PB 235, PB 330 e PB 217, submetidos a nove sistemas de sangria: 1/2S d/3.ET 2,5\% 8/y;

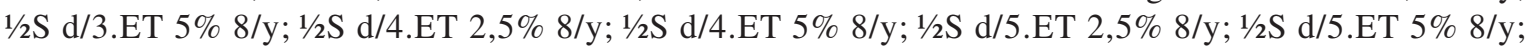
$1 / 2 \mathrm{~S} \mathrm{~d} / 7$. ET 2,5\% 8/y; $1 / 2 \mathrm{~S} \mathrm{~d} / 7$. ET $5 \%$ 8/y e $1 / 2 \mathrm{~S}$ d/2 (testemunha). As variáveis avaliadas foram: perímetro do caule, produção de borracha seca, secamento do painel; foi avaliada, também, a viabilidade econômica. Os resultados mostram a superioridade econômica dos sistemas $1 \frac{1}{2} \mathrm{~S}$ d/5.ET 2,5\% 8/y e 1/2S d/7.ET 5\% 8/y, para o clone PB 260; $1 / 2 \mathrm{~S} \mathrm{~d} / 7$.ET 2,5\% 8/y para o clone PB 235; e 1/2S d/3.ET 2,5\% 8/y e 1/2S d/3.ET 5\% 8/y, para os clones PB 330 e PB 217, comparados com a testemunha. A menor incidência de secamento do painel foi observada no sistema $1 / 2 \mathrm{~S}$ d/7.ET 5\% 8/y, exceto para o clone PB 235.
\end{abstract}

Termos para indexação: Hevea brasiliensis, borracha natural, estimulação química, secamento do painel, economia.

\section{Rubber tree exploitation systems utilized in Asian clones Prang Besar, in the West of São Paulo, Brazil}

\begin{abstract}
The objective of this work was to evaluate the yield performance, and economic and physiologic aspects of four Asian clones Prang Besar of rubber tree [Hevea brasiliensis (Willd. ex A. Juss.) Muell. Arg.], in different tapping systems. The trial was placed at Santa Gilda farm, City of Guararapes, São Paulo State, Brazil, in a randomized block design with split-plot in time. The main treatments were PB 260, PB 235, PB 330 and PB 217

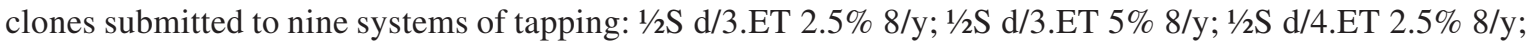

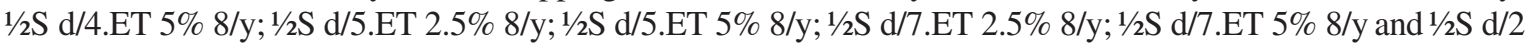
(check). The variables evaluated were: girth increment, dry rubber production, brown bast; it was also evaluated the economic viability. Results indicated the economical superiority of the $1 / 2 \mathrm{~S} d / 5$.ET $2.5 \% 8 / \mathrm{y}$ and $1 / 2 \mathrm{~S} \mathrm{~d} / 7$.ET 5\% 8/y systems in PB 260 clone; $1 \frac{1}{2} \mathrm{~S}$ d/7.ET $2.5 \%$ 8/y system in PB 235 clone; and $1 \frac{1}{2} \mathrm{~S} d / 3$.ET $2.5 \%$ 8/y and $1 / 2 \mathrm{~S}$ d/3.ET 5\% 8/y in PB 330 and PB 217 clones, compared with the check. The smallest incidence of panel desiccation was observed in $1 / 2 \mathrm{~S}$ d/7.ET 5\% 8/y system, except for PB 235 clone.
\end{abstract}

Index terms: Hevea brasiliensis, natural rubber, chemical stimulation, brown bast, economy.

\section{Introdução}

A seringueira [Hevea brasiliensis (Willd. ex A. Juss.) Muell. Arg.], pertencente à família Euphorbiaceae, é uma espécie de grande importância por ser a principal fonte de borracha natural explotada no mundo. Hoje, o Brasil responde por aproximadamente $1 \%$ da produção mundial de borracha natural, porém apesar dessa pequena contribuição, o setor tem grande importância no país, o que pode ser confirmado pela presença de inúmeras indústrias de transformação, especialmente a pneumática, e por um consumo que ainda está longe de ser atendido pela produção nacional. Atualmente, o deficit de borracha natural chega a quase $179 \mathrm{mil}$ toneladas, o que justifica a necessidade de expansão da heveicultura nacional (IRSG, 2007). No contexto brasileiro, o Estado de São Paulo é o primeiro produtor de borracha natural desde 1995, com uma produção 
aproximada, em 2004, de 95 mil toneladas de látex coagulado (IBGE, 2007).

Nos últimos anos, tendências de aumento no custo de produção de borracha natural levaram a uma busca continuada de formas de explotação, que proporcionam redução desses custos. De acordo com Gonçalves et al. (2000), a explotação do seringal, operação popularmente conhecida como sangria, é um ponto importante na heveicultura, e representa $60 \%$ dos custos totais da borracha produzida, além de determinar a vida útil das árvores e a produtividade.

$\mathrm{O}$ incremento de produtividade, pela adoção de métodos apropriados de explotação, é uma das soluções para se alcançar maiores ganhos líquidos (Rajagopal et al., 2004). De acordo com Gonçalves et al. (2000), a mão-de-obra representa a maior parte dos custos de produção e, atualmente, é uma das principais dificuldades encontradas na heveicultura paulista, pois há falta de mão-de-obra qualificada.

O uso de estimulante para aumento da produção, prática que tem sido utilizada com freqüência nos seringais de cultivo há bastante tempo, é a alternativa adotada para a redução na freqüência de sangria e, conseqüentemente, redução nos custos de produção. Experimentos têm demonstrado que sistemas de baixa freqüência de sangria com o uso de ethefon possibilitam boas produções com economia nos gastos com mãode-obra (Virgens Filho et al., 1986). De acordo com Conduru Neto (1986), a utilização de substâncias estimulantes possibilita o aumento da produção de látex, com a adoção de menores freqüências de sangria, e permite atingir os dois fatores básicos de produção: fisiológico e econômico.

Diversos autores, entre eles Bernardes et al. (1995), Premakumari et al. (1997) e Usha Nair et al. (2004), têm relatado que outro grande problema de importância, no contexto da heveicultura, é o distúrbio fisiológico conhecido como seca-do-painel (brown bast ou tapping panel dryness). Esse distúrbio ocorre na fase de sangria, bloqueia o fluxo de látex contido no interior da casca do caule, e pode causar uma total ou parcial redução da produção (Gonçalves et al., 2001). Provavelmente, uma das causas é a adoção de sistemas de explotação com alta freqüência de sangria e altas concentrações de estimulante, porém é importante frisar que as causas primárias desse distúrbio ainda não foram elucidadas.

De acordo com Toledo \& Ghilardi (2000), indicadores técnicos, econômicos e financeiros são, sem dúvida, ferramentas indispensáveis para a tomada de decisão do empresário rural. Portanto, a introdução dos clones de seringueira exige uma definição sobre os sistemas de explotação a serem adotados, levando-se em conta esses indicadores, pois segundo Bernardes et al. (2000), existe variação acentuada no comportamento de cada material vegetal, quando submetido a diferentes sistemas de explotação.

O objetivo do trabalho foi identificar os melhores sistemas de explotação do látex, para cada um dos quatro clones asiáticos Prang Besar de seringueira, na região Oeste do Estado de São Paulo, e relacioná-los a aspectos de viabilidade econômica e aspectos fisiológicos, como a menor incidência de seca-do-painel e maior produtividade.

\section{Material e Métodos}

O experimento foi conduzido na Fazenda Santa Gilda, pertencente ao grupo Rodobens Agrícola e Pecuária Ltda., no Município de Guararapes, região Oeste do Estado de São Paulo, a $21^{\circ} 20^{\prime} \mathrm{S}, 50^{\circ} 50^{\prime} \mathrm{W}$ e altitude de $560 \mathrm{~m}$. De acordo com a classificação de Köppen, o clima da região é do tipo Aw, com precipitação média anual de $1.270,8 \mathrm{~mm}$, temperatura média anual de $24^{\circ} \mathrm{C}$, temperatura média do mês mais frio de $20,2^{\circ} \mathrm{C}$ e temperatura média do mês mais quente de $26^{\circ} \mathrm{C}$.

$\mathrm{O}$ plantio dos clones asiáticos de seringueira [Hevea brasiliensis (Willd. ex A. Juss.) Muell. Arg.] foi realizado em 1992, no espaçamento 8x2,5 m, em um Argissolo Vermelho eutrófico, arênico, profundo e bem drenado (Prado, 2003). Todos os tratos culturais ministrados ao experimento seguiram as recomendações técnicas para a cultura no Estado de São Paulo, de acordo com Gonçalves et al. (2001).

O delineamento experimental utilizado foi o de blocos ao acaso, com parcelas subdivididas no tempo e quatro repetições, de acordo com Steel \& Torrie (1980). Os tratamentos principais, alocados em parcelas de 0,25 ha, em cada repetição, no total de 1 ha, foram os clones: PB 260, PB 235, PB 330 e PB 217. Os tratamentos secundários, alocados nas subparcelas compostas por 12 plantas, com exceção da testemunha, que foi composta por 24 plantas, são mostrados na Tabela 1, onde: $1 / 2 \mathrm{~S}$ é a sangria em meia espiral; $\mathrm{d} / 2, \mathrm{~d} / 3$, d/4, d/5 e d/7 são sangrias a cada 2, 3, 4, 5 e 7 dias, respectivamente; $11 \mathrm{~m} / \mathrm{y}$ são árvores que sangraram durante 11 meses por ano; ET é o ethefon; Pa La é a aplicação do estimulante no painel de sangria, sobre a 
canaleta com cernambi; 8/y é a aplicação do estimulante oito vezes por ano.

O registro da produtividade de borracha foi efetuado mensalmente pelo látex coagulado naturalmente nas tigelas. Houve adição de ácido acético a 5\%, apenas em dias com ocorrência de chuva após a sangria. A massa total mensal de todas as árvores, de cada subtratamento, foi dividida pelo número total de coágulos, e os resultados foram expressados em gramas de borracha seca por corte por árvore. Com esses dados, estimou-se a produção por hectare ao ano por clone, em cada sistema de sangria, tendo-se adotado 140, 104, 78,62 e 52 cortes por ano, nas frequiências d/2, d/3, d/4, $\mathrm{d} / 5$ e d/7, respectivamente. Na estimativa dos dados de produção de borracha, extrapolados para hectare por ano, adotou-se, nos cálculos, estandes de 240, 340, 380 e 400 árvores em sangria, por hectare, no primeiro, segundo, terceiro, quarto e quinto anos, respectivamente (Embrapa, 1987).

Fez-se o levantamento da incidência de árvores secas, ao final de cinco anos de avaliação. Os painéis secos foram avaliados em cada sistema de sangria para cada clone, e foi calculada a porcentagem de painéis secos, de acordo com o número de plantas em cada sistema de sangria.

Foram realizadas análises anuais com média das parcelas, para a avaliação do vigor das árvores, expressadas em perímetro do caule. As mensurações foram conduzidas a 1,20 m do calo de enxertia, durante cinco anos de avaliação, tendo-se avaliado cinco plantas por parcela.

Para a análise econômica, foram estudados parâmetros: receita bruta, salários, insumos, materiais agrícolas e, assim, foram determinados o custo operacional efetivo (COE) na produção, a receita líquida e a rentabilidade, para cada clone, por sistema de explotação. O COE é composto pela soma das despesas diretas, que implicam desembolso real pelo produtor (Toledo \& Ghilardi, 2000), portanto, ressalte-se, não se trata de um custo total de produção, visto que não foram incluídas: a remuneração ao capital envolvido (fixo ou circulante), a retribuição ao fator terra, a remuneração ao trabalho do empresário e a depreciação dos equipamentos.

Neste trabalho, o custo operacional efetivo foi contemplado no período da sangria e não da cultura, pois não foram consideradas as despesas com a implantação que foi comum aos diferentes clones e tratamentos. Foram anotados os gastos anuais com insumos (ethefon e ácido acético), materiais agrícolas (tigelas, bicas, arame, faca-de-sangria e esmeril), para eventuais reposições e salários, juntamente com encargos sociais, tendo-se considerado que um seringueiro sangra 800 árvores por dia. A receita líquida correspondeu à diferença entre a receita bruta e o custo operacional efetivo. A rentabilidade dos sistemas de sangria, de cada clone, foi calculada em relação à testemunha e expressada em porcentagem.

Os dados de produtividade foram submetidos à análise de variância, pelo teste $\mathrm{F}$, a $1 \%$ de probabilidade. No esquema de parcelas subdivididas no tempo, os tratamentos (clones) constituíram as parcelas, os subtratamentos (sistemas de sangria) constituíram as subparcelas e os subsubtramentos (anos) constituíram as subsubparcelas. Realizou-se a análise do desdobramento, na interação de clones x sistemas de sangria a $1 \%$ de probabilidade, e procedeu-se ao teste de Tukey a $1 \%$ de probabilidade para comparação das médias.

Tabela 1. Subtratamentos utilizados no experimento com quatro clones de seringueiras, em cinco anos de avaliação, de acordo com o sistema internacional de notação para a sangria da seringueira.

\begin{tabular}{|c|c|c|c|c|c|c|c|}
\hline \multirow[t]{2}{*}{ Subtratamento $^{(1)}$} & \multicolumn{3}{|c|}{ Notação de sangria } & \multicolumn{4}{|c|}{ Notação de estimulação } \\
\hline & $\begin{array}{l}\text { Comprimento do } \\
\text { corte }\end{array}$ & $\begin{array}{c}\text { Freqüência de } \\
\text { sangria }\end{array}$ & $\begin{array}{c}\text { Periodicidade de } \\
\text { sangria }\end{array}$ & $\begin{array}{l}\text { Ingrediente ativo } \\
\text { do estimulante }\end{array}$ & $\begin{array}{c}\text { Concentração do } \\
\text { estimulante }\end{array}$ & $\begin{array}{l}\text { Método de } \\
\text { aplicação }\end{array}$ & $\begin{array}{c}\text { Freqüência de } \\
\text { estimulação }\end{array}$ \\
\hline 1 & $1 / 2 \mathrm{~S}$ & $\mathrm{~d} / 2$ & $11 \mathrm{~m} / \mathrm{y}$ & - & - & - & - \\
\hline 2 & $1 / 2 \mathrm{~S}$ & $d / 3$ & $11 \mathrm{~m} / \mathrm{y}$ & ET & $2,5 \%$ & $\mathrm{~Pa} \mathrm{La}$ & $8 / y$ \\
\hline 3 & $1 / 2 \mathrm{~S}$ & $d / 3$ & $11 \mathrm{~m} / \mathrm{y}$ & ET & $5,0 \%$ & $\mathrm{~Pa} \mathrm{La}$ & $8 / y$ \\
\hline 4 & $1 / 2 \mathrm{~S}$ & $\mathrm{~d} / 4$ & $11 \mathrm{~m} / \mathrm{y}$ & ET & $2,5 \%$ & $\mathrm{~Pa} \mathrm{La}$ & $8 / y$ \\
\hline 5 & $1 / 2 \mathrm{~S}$ & $\mathrm{~d} / 4$ & $11 \mathrm{~m} / \mathrm{y}$ & ET & $5,0 \%$ & $\mathrm{~Pa} \mathrm{La}$ & $8 / y$ \\
\hline 6 & $1 / 2 \mathrm{~S}$ & $d / 5$ & $11 \mathrm{~m} / \mathrm{y}$ & ET & $2,5 \%$ & $\mathrm{~Pa} \mathrm{La}$ & $8 / y$ \\
\hline 7 & $1 / 2 \mathrm{~S}$ & $d / 5$ & $11 \mathrm{~m} / \mathrm{y}$ & ET & $5,0 \%$ & $\mathrm{~Pa} \mathrm{La}$ & $8 / y$ \\
\hline 8 & $1 / 2 \mathrm{~S}$ & $\mathrm{~d} / 7$ & $11 \mathrm{~m} / \mathrm{y}$ & ET & $2,5 \%$ & $\mathrm{~Pa} \mathrm{La}$ & $8 / y$ \\
\hline 9 & $1 / 2 \mathrm{~S}$ & $\mathrm{~d} / 7$ & $11 \mathrm{~m} / \mathrm{y}$ & ET & $5,0 \%$ & $\mathrm{~Pa} \mathrm{La}$ & $8 / y$ \\
\hline
\end{tabular}

${ }^{(1)}$ Subtratamento 1 corresponde à testemunha. 


\section{Resultados e Discussão}

$\mathrm{Na}$ análise de variância conjunta das médias de produtividade de borracha seca, houve diferença significativa quanto aos clones, sistemas de sangria e em relação à interação clones x sistemas de sangria (Tabela 2). A interação clones $\mathrm{x}$ sistemas de sangria $\mathrm{x}$ anos também foi significativa, porém por ser de interesse secundário, não foi estudada. Os resultados de coeficiente de variação estão dentro dos limites observados por Gonçalves et al. (2000), para a cultura da seringueira.

Tabela 2. Quadrados médios da análise de variância conjunta dos dados de produtividade de borracha seca $\left(\mathrm{kg} \mathrm{ha}^{-1} \mathrm{ano}^{-1}\right)$, de cinco anos de avaliação de quatro clones de seringueira, sob nove sistemas de sangria.

\begin{tabular}{lrc}
\hline Fonte de variação & GL & Quadrado médio \\
\hline Blocos & 3 & $627.181,47^{\text {ns }}$ \\
Clones (A) & 3 & $3.649 .485,54^{* *}$ \\
Resíduo (a) & 9 & $113.678,09$ \\
Sistemas de sangria (B) & 8 & $2.030 .596,42^{* *}$ \\
A x B & 24 & $116.388,83^{* *}$ \\
Resíduo (b) & 96 & 507,90 \\
Anos (C) & 4 & $15.075 .323,57^{* *}$ \\
A x C & 12 & $1.108 .044,79^{* *}$ \\
B x C & 32 & $139.225,43^{* *}$ \\
A x B x C & 96 & $40.155,38^{* *}$ \\
Resíduo (c) & 432 & $8.967,74$ \\
\hline Total & 719 & \\
\hline Média geral & 1.240 & \\
CV (\%) (A) & 9,06 & \\
CV (\%) (B) & 1,82 & \\
CV (\%) (C) & 7,64 & \\
\hline
\end{tabular}

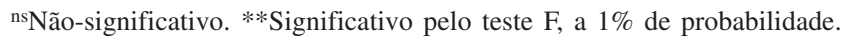

Os resultados da análise anual das médias de produtividade de borracha mostram que os clones só apresentaram diferença significativa entre si, a partir do quarto ano de produção (Tabela 3). A interação clones $\mathrm{x}$ sistemas de sangria foi significativa em todos os anos. $\mathrm{Na}$ análise do desdobramento, os sistemas de sangria, em todos os clones e em todos os anos, apresentaram diferença significativa pelo teste $\mathrm{F}$.

Na Tabela 4 estão descritos o perímetro médio anual do caule e seus incrementos porcentuais, relativos aos cinco anos de avaliação e referentes à média das parcelas. De acordo com Gonçalves et al. (2006), a produção de borracha não está necessariamente correlacionada ao incremento do perímetro do caule. O clone que apresentou maior perímetro na abertura do painel de sangria foi o PB 260, entretanto, esse clone tem apresentado baixa taxa de crescimento durante todo o período de desenvolvimento, em experimentos realizados em Votuporanga, SP (SAA, 2006). Precocidade é um caráter desejável, pois permite diminuir o período improdutivo das árvores. O clone que apresentou maior incremento médio, nos cinco anos de avaliação, foi o PB 217 e esse caráter é importante, pois diminui a probabilidade de quebra das árvores por ventos (Gonçalves et al., 1990).

Os dados obtidos em relação à seca-do-painel mostram o comportamento diferente dos clones, o que confirma a teoria que a incidência desse distúrbio é dependente do genótipo da planta, além da influência de fatores ambientais (Tabela 5); houve mais de 5\% de incidência da seca-do-painel em plantas sob alguns sistemas de sangria, e foi necessária uma revisão do método de explotação adotado, de acordo com a recomendação do Instituto Agronômico (Gonçalves

Tabela 3. Quadrados médios da análise de variância referente às médias de produtividade de borracha seca $\left(\mathrm{kg} \mathrm{ha}^{-1} \mathrm{ano}^{-1}\right)$ de cinco anos, de quatro clones de seringueira sob nove sistemas de sangria.

\begin{tabular}{|c|c|c|c|c|c|c|c|}
\hline \multirow[t]{2}{*}{ Fonte de variação } & \multirow[t]{2}{*}{ GL } & \multicolumn{6}{|c|}{ Sangrias anuais } \\
\hline & & $1^{\circ}$ ano & $2^{\underline{0}}$ ano & $3^{\circ}$ ano & $4^{\circ}$ ano & $5^{0}$ ano & Média \\
\hline Blocos & 3 & $25.882,52^{\text {ns }}$ & $125.542,69^{\mathrm{ns}}$ & $213.069,66^{\mathrm{ns}}$ & $156.844,93^{\mathrm{ns}}$ & $239.149,41^{\mathrm{ns}}$ & $125.438,03^{\mathrm{ns}}$ \\
\hline Clones (A) & 3 & $175.228,06^{\mathrm{ns}}$ & $728.765,17^{\text {ns }}$ & $656.764,43^{\mathrm{ns}}$ & $2.143 .063,55^{* *}$ & $4.377 .843,46^{* *}$ & $729.891,72 * *$ \\
\hline Resíduo (a) & 9 & $31.151,29$ & $42.416,23$ & $102.405,58$ & $119.855,82$ & $92.265,54$ & $22.735,81$ \\
\hline Sistemas de sangria (B) & 8 & $151.305,88^{* *}$ & $380.049,33 * *$ & $485.331,22 * *$ & $757.702,47 * *$ & $813.109,25^{* *}$ & $406.120,52 * *$ \\
\hline $\mathrm{A} \times \mathrm{B}$ & 24 & $6.353,21^{* *}$ & $22.779,27 * *$ & $27.058,54^{* *}$ & $75.659,08^{* *}$ & $145.160,26^{* *}$ & $23.277,79 * *$ \\
\hline Resíduo (b) & 96 & 79,51 & 209,25 & 264,38 & 459,51 & 582,69 & 101,58 \\
\hline Total & 143 & & & & & & \\
\hline Média geral & & 663,56 & $1.428,75$ & $1.386,33$ & $1.369,87$ & $1.352,13$ & $1.240,13$ \\
\hline $\mathrm{CV}(\%)(\mathrm{A})$ & & 8,87 & 8,80 & 7,69 & 8,42 & 7,49 & 4,05 \\
\hline $\mathrm{CV}(\%)(\mathrm{B})$ & & 1,34 & 1,01 & 1,17 & 1,56 & 1,79 & 0,81 \\
\hline
\end{tabular}

${ }^{n s}$ Não-significativo. $* *$ Significativo a $1 \%$ de probabilidade, pelo teste $\mathrm{F}$. 
et al., 2001). O clone PB 235 apresentou maior susceptibilidade a esse distúrbio, e os dados encontrados para esse clone concordam com os obtidos pelo RRIM (1989), cuja classificação para esse caráter secundário é "abaixo da média". Segundo Gonçalves et al. (2001), a seca-do-painel tornou-se um problema de grande importância, nesse clone, na Costa do Marfim. Todos os clones estudados no sistema de sangria $1 / 2 \mathrm{~S} d / 2$ (testemunha) apresentaram baixa incidência, o que pode ser explicado pela teoria de que a estimulação da produção pode tornar as plantas mais suscetíveis. Foi observada, também, menor incidência nas plantas sob o sistema $1 \frac{1}{2} \mathrm{~S}$ d/7.ET $5 \%$ 8/y, com exceção no clone PB 235, o que reforça a idéia de que uma combinação adequada de sistemas com baixa frequiência de sangria e estimulação diminui a possibilidade de ocorrência desse distúrbio.

As médias de produtividade de borracha seca indicam que, em cinco anos de sangria, a maioria dos clones respondeu bem ao sistema $1 \frac{1}{2} \mathrm{~S} d / 3$, com estimulação de ethefon a 2,5 e $5 \%$ (Tabela 6). O clone PB 235 não respondeu à estimulação, e o sistema $1 / 2 \mathrm{~S} d / 2$ mostrou superioridade em relação à média de produtividade. Esse resultado corrobora os relatos de Bernardes et al. (1995) e Gonçalves et al. (2000), que explicam a fraca resposta desse clone aos sistemas de explotação com estimulação. De acordo com Conduru Neto (1986), no entanto, o clone PB 235 é considerado altamente produtivo, mas como não responde à estimulação, não é indicado para regiões onde a mão-de-obra seja fator limitante.

A análise econômica dos diferentes sistemas de sangria, dos quatro clones de seringueira, com base na produtividade média de cinco anos de avaliação é apresentada na Tabela 6. Observa-se que houve redução de $67 \%$ sobre o item de despesa mão-de-obra, na frequiência de sangria d/7, em relação à frequiência d/2, para todos os clones. De acordo com Pinheiro et al. (2002), nessa frequiência, um seringueiro pode sangrar até 7 mil árvores, o que confere redução significativa nos custos operacionais. Observa-se que, para o clone PB 260, o sistema que proporcionou maior rentabilidade foi o $1 / 2 \mathrm{~S} \mathrm{~d} / 3 \mathrm{ET}$. 2,5\% 8/y, entretanto, no mesmo clone, foi observada alta incidência de seca-do-painel (8\%) o que o inviabiliza do ponto de vista fisiológico; sugere-se, portanto, $\mathrm{o}^{1 / 2 \mathrm{~S} d} \mathrm{~d} / 5$. ET $2,5 \% 8 / \mathrm{y}$ ou o $1 \frac{1}{2} \mathrm{~S}$ d/7.ET $5 \% 8 / \mathrm{y}$, nos quais não foi observada a incidência desse distúrbio, e que apresentam rentabilidade satisfatória. Em razão da baixa resposta do clone PB 235 à estimulação, os sistemas de sangria com estimulação apresentaram baixa

Tabela 4. Perímetro do caule e incrementos de cinco anos de sangria, referentes a quatro clones de seringueira ${ }^{(1)}$.

\begin{tabular}{|c|c|c|c|c|c|c|c|c|}
\hline \multirow[t]{2}{*}{ Ano } & \multicolumn{4}{|c|}{ Perímetro $(\mathrm{cm})$} & \multicolumn{4}{|c|}{ Incremento (\%) } \\
\hline & PB 260 & PB 235 & PB 330 & PB 217 & PB 260 & PB 235 & PB 330 & PB 217 \\
\hline $1^{(2)}$ & 61,25 & 50,80 & 52,20 & 45,20 & - & - & - & - \\
\hline 2 & 63,25 & 52,80 & 53,60 & 47,80 & 3,27 & 3,94 & 2,68 & 5,75 \\
\hline 3 & 65,25 & 54,20 & 54,80 & 49,00 & 3,16 & 2,65 & 2,24 & 2,51 \\
\hline 4 & 66,25 & 55,20 & 55,60 & 50,60 & 1,53 & 1,85 & 1,46 & 3,27 \\
\hline 5 & 69,00 & 58,20 & 59,00 & 52,80 & 4,15 & 5,43 & 6,12 & 4,35 \\
\hline Média & 65,00 & 54,24 & 55,04 & 49,08 & 3,03 & 3,47 & 3,12 & 3,97 \\
\hline Desvio-padrão & 2,95 & 2,76 & 2,56 & 2,87 & & & & \\
\hline
\end{tabular}

${ }^{(1)}$ Valores referem-se à média das parcelas. ${ }^{(2)}$ Ano 1 corresponde à abertura do painel de sangria.

Tabela 5. Incidência de secamento do painel, em quatro clones de seringueira, no quinto ano de avaliação, em diferentes sistemas de sangria.

\begin{tabular}{|c|c|c|c|c|c|c|c|c|}
\hline \multirow[t]{2}{*}{ Sistema de sangria } & \multicolumn{4}{|c|}{ Número total de árvores sangradas } & \multicolumn{4}{|c|}{ Porcentagem de árvores com seca-do-painel $^{(1)}$} \\
\hline & PB 260 & PB 235 & PB 330 & PB 217 & PB 260 & PB 235 & PB 330 & PB 217 \\
\hline $1 / 2 \mathrm{~S} \mathrm{~d} / 2$ (test.) & 97 & 95 & 97 & 92 & 3,0 & 3,1 & 3,0 & 3,2 \\
\hline $1 / 2 \mathrm{~S} \mathrm{~d} / 3 . \operatorname{ET} 2,5 \% 8 / \mathrm{y}$ & 46 & 47 & 49 & 43 & 8,0 & 6,0 & 2,0 & 0,0 \\
\hline $1 / 2 \mathrm{~S} \mathrm{~d} / 3 . \mathrm{ET} 5 \% 8 / \mathrm{y}$ & 46 & 44 & 49 & 46 & 8,0 & 12,0 & 2,0 & 2,1 \\
\hline $1 / 2 \mathrm{~S} \mathrm{~d} / 4$.ET $2,5 \% 8 / y$ & 47 & 49 & 49 & 46 & 6,0 & 2,0 & 0,0 & 2,1 \\
\hline $1 / 2 \mathrm{~S} \mathrm{~d} / 4$.ET $5 \% 8 / \mathrm{y}$ & 48 & 46 & 45 & 49 & 4,0 & 8,0 & 10,0 & 2,0 \\
\hline $1 / 2 \mathrm{~S} \mathrm{~d} / 5$.ET $2,5 \% 8 / \mathrm{y}$ & 50 & 48 & 47 & 45 & 0,0 & 4,0 & 2,1 & 4,3 \\
\hline $1 / 2 \mathrm{~S} \mathrm{~d} / 5$.ET $5 \% 8 / \mathrm{y}$ & 46 & 44 & 48 & 44 & 8,0 & 12,0 & 2,0 & 0,0 \\
\hline $1 / 2 \mathrm{~S} \mathrm{~d} / 7 . \mathrm{ET} 2,5 \% 8 / \mathrm{y}$ & 47 & 50 & 49 & 39 & 6,0 & 0,0 & 2,0 & 9,3 \\
\hline $1 / 2 \mathrm{~S} \mathrm{~d} / 7$.ET $5 \% 8 / \mathrm{y}$ & 50 & 47 & 50 & 42 & 0,0 & 6,0 & 0,0 & 2,3 \\
\hline
\end{tabular}

(1)Porcentagem de seca-do-painel em relação ao número total de plantas, em cada subtratamento. 
rentabilidade, em relação à testemunha, como nos sistemas $1 / 2 \mathrm{~S} d / 4$ e $1 / 2 \mathrm{~S} d / 5$, cujo ganho foi menor que o sistema $1 / 2 \mathrm{~S} d / 2$. Para esse clone, apesar da fraca resposta ao estimulante, recomenda-se o sistema $1 / 2 \mathrm{~S} \mathrm{~d} / 7$.ET $2,5 \% 8 / \mathrm{y}$, pela ausência de incidência de seca-do-painel, além de apresentar superioridade de $14 \%$, em termos de rentabilidade, em relação à testemunha. Para os clones PB 330 e PB 217, a maior rentabilidade, observada em $1 / 2 \mathrm{~S}$ d/3 com estimulação de ethefon a 2,5 e $5 \%$, somada à baixa incidência de seca-do-painel, é um bom indicativo para adoção desse sistema.
Apesar da significativa rentabilidade financeira alcançada nos sistemas com maiores freqüências de sangria, para os clones PB 330 e PB 217, pode-se esperar uma situação favorável para os sistemas com menores frequiências de sangria $(\mathrm{d} / 7)$, à medida que ocorra aumento no custo da mão-de-obra, ou possível diminuição dos preços da borracha, uma vez que esses representam redução de mais da metade do custo com salários, em relação aos sistemas de alta freqüência. Além disso, é de se esperar que esses sistemas de sangria proporcionem uma maior vida produtiva das árvores.

Tabela 6. Produtividade de borracha seca e análise econômica de diferentes sistemas de sangria de quatro clones de seringueira, com base na média de produtividade de borracha seca de cinco anos de avaliação, no Município de Guararapes, SP.

\begin{tabular}{|c|c|c|c|c|c|c|c|c|c|}
\hline Clone & Sistema de sangria & $\begin{array}{l}\text { Produtividade } \\
\left(\mathrm{kg} \mathrm{ha}^{-1} \mathrm{ano}^{-1}\right)^{(1)}\end{array}$ & $\begin{array}{l}\text { Receita bruta } \\
(\mathrm{R} \$)^{(2)}\end{array}$ & $\begin{array}{l}\text { Mão-de-obra } \\
(\mathrm{R} \$)^{(3)}\end{array}$ & $\begin{array}{l}\text { Insumos } \\
(\mathrm{R} \$)^{(4)}\end{array}$ & $\begin{array}{c}\text { Materiais } \\
\text { agrícolas }(\mathrm{R} \$)^{(5)}\end{array}$ & $\begin{array}{c}\text { Custos operacionais } \\
\text { efetivos (R\$) }\end{array}$ & $\begin{array}{c}\text { Receita } \\
\text { líquida (R\$) }\end{array}$ & $\begin{array}{c}\text { Rentabilidade } \\
(\%)^{(6)}\end{array}$ \\
\hline \multirow[t]{8}{*}{ PB 260} & $1 / 2 \mathrm{~S} \mathrm{~d} / 2$ (testemunha) & $1.201,82 \mathrm{~b}$ & $4.206,38$ & $2.112,50$ & 11,03 & 35,00 & $2.158,53$ & $2.047,85$ & 100 \\
\hline & $1 / 2 \mathrm{~S} \mathrm{~d} / 3$. ET $2,5 \% 8 / y$ & $1.246,90 \mathrm{a}$ & $4.364,16$ & $1.408,33$ & 129,31 & 35,00 & $1.572,64$ & $2.791,52$ & 136 \\
\hline & $1 / 2 \mathrm{~S} \mathrm{~d} / 3$. ET $5 \% 8 / \mathrm{y}$ & $1.210,75 b$ & $4.237,64$ & $1.408,33$ & 247,58 & 35,00 & $1.690,91$ & $2.546,73$ & 124 \\
\hline & $1 / 2 \mathrm{~S} \mathrm{~d} / 4$.ET $2,5 \% 8 / \mathrm{y}$ & $1.046,93 \mathrm{c}$ & $3.664,26$ & $1.056,25$ & 129,31 & 35,00 & $1.220,56$ & $2.443,70$ & 119 \\
\hline & $1 / 2 \mathrm{~S} \mathrm{~d} / 4$.ET $5 \% 8 / \mathrm{y}$ & $987,46 \mathrm{e}$ & $3.456,09$ & $1.056,25$ & 247,58 & 35,00 & $1.338,83$ & $2.117,27$ & 103 \\
\hline & $1 / 2 \mathrm{~S} \mathrm{~d} / 5$.ET $2,5 \% 8 / \mathrm{y}$ & $1.016,07 \mathrm{~d}$ & $3.556,25$ & 845,00 & 129,31 & 35,00 & $1.009,31$ & $2.546,94$ & 124 \\
\hline & $1 / 2 \mathrm{~S} \mathrm{~d} / 5 . \mathrm{ET} 5 \% 8 / \mathrm{y}$ & $865,80 \mathrm{~g}$ & $3.030,29$ & 845,00 & 247,58 & 35,00 & $1.127,58$ & $1.902,71$ & 93 \\
\hline & $1 / 2 \mathrm{~S} \mathrm{~d} / 7$. ET $5 \% 8 / \mathrm{y}$ & $991,05 \mathrm{de}$ & $3.468,66$ & 704,17 & 247,58 & 35,00 & 986,74 & $2.481,92$ & 121 \\
\hline \multirow[t]{8}{*}{ PB 235} & $1 / 2 \mathrm{~S} \mathrm{~d} / 2$ (testemunha) & $1.473,81 \mathrm{a}$ & $5.158,32$ & $2.112,50$ & 11,03 & 35,00 & 158,53 & $2.999,79$ & 100 \\
\hline & $1 / 2 \mathrm{~S} \mathrm{~d} / 3 . \operatorname{ET} 2,5 \% 8 / \mathrm{y}$ & $1.407,47 \mathrm{~b}$ & $4.926,16$ & $1.408,33$ & 129,31 & 35,00 & $1.572,64$ & $3.353,52$ & 112 \\
\hline & $1 / 2 \mathrm{~S} \mathrm{~d} / 3 . \mathrm{ET} 5 \% 8 / \mathrm{y}$ & $1.431,93 b$ & $5.011,77$ & $1.408,33$ & 247,58 & 35,00 & $1.690,91$ & $3.320,86$ & 111 \\
\hline & $1 / 2 \mathrm{~S} \mathrm{~d} / 4$.ET $2,5 \% 8 / \mathrm{y}$ & $1.188,47 \mathrm{~d}$ & $4.159,65$ & $1.056,25$ & 129,31 & 35,00 & $1.220,56$ & $2.939,10$ & 98 \\
\hline & $1 / 2 \mathrm{~S} \mathrm{~d} / 4$.ET $5 \% 8 / \mathrm{y}$ & $1.125,36 \mathrm{e}$ & $3.938,77$ & $1.056,25$ & 247,58 & 35,00 & $1.338,83$ & $2.599,95$ & 87 \\
\hline & $1 / 2 \mathrm{~S} \mathrm{~d} / 5$.ET $2,5 \% 8 / y$ & $1.098,17 \mathrm{f}$ & $3.843,59$ & 845,00 & 129,31 & 35,00 & $1.009,31$ & $2.834,29$ & 94 \\
\hline & $1 / 2 \mathrm{~S} \mathrm{~d} / 5 . \mathrm{ET} 5 \% 8 / \mathrm{y}$ & $1.022,16 \mathrm{~g}$ & $3.577,58$ & 845,00 & 247,58 & 35,00 & $1.127,58$ & $2.450,00$ & 82 \\
\hline & $1 / 2 \mathrm{~S} \mathrm{~d} / 7$. ET $5 \% 8 / \mathrm{y}$ & $1.230,09 \mathrm{c}$ & $4.305,32$ & 704,17 & 247,58 & 35,00 & 986,74 & $3.318,57$ & 111 \\
\hline \multirow[t]{9}{*}{ PB 330} & $1 / 2 \mathrm{~S} \mathrm{~d} / 2$ (testemunha) & $1.384,56 \mathrm{c}$ & $4.845,95$ & $2.112,50$ & 11,03 & 35,00 & $2.158,53$ & $2.687,41$ & 100 \\
\hline & $1 / 2 \mathrm{~S} \mathrm{~d} / 3$. ET $2,5 \% 8 / y$ & $1.453,33 b$ & $5.086,65$ & $1.408,33$ & 129,31 & 35,00 & $1.572,64$ & $3.514,01$ & 131 \\
\hline & $1 / 2 \mathrm{~S} \mathrm{~d} / 3 . \mathrm{ET} 5 \% 8 / \mathrm{y}$ & $1.528,50 \mathrm{a}$ & $5.349,74$ & $1.408,33$ & 247,58 & 35,00 & $1.690,91$ & $3.658,83$ & 136 \\
\hline & $1 / 2 \mathrm{~S} \mathrm{~d} / 4$.ET $2,5 \% 8 / y$ & $1.228,46 \mathrm{e}$ & $4.299,60$ & $1.056,25$ & 129,31 & 35,00 & $1.220,56$ & $3.079,05$ & 115 \\
\hline & $1 / 2 \mathrm{~S} \mathrm{~d} / 4$. ET $5 \% 8 / \mathrm{y}$ & $1.268,41 \mathrm{~d}$ & $4.439,45$ & $1.056,25$ & 247,58 & 35,00 & $1.338,83$ & $3.100,62$ & 115 \\
\hline & $1 / 2 \mathrm{~S} \mathrm{~d} / 5 . \mathrm{ET} 2,5 \% 8 / \mathrm{y}$ & $1.107,69 \mathrm{~g}$ & $3.876,92$ & 845,00 & 129,31 & 35,00 & $1.009,31$ & $2.867,62$ & 107 \\
\hline & $1 / 2 \mathrm{~S} \mathrm{~d} / 5 . \mathrm{ET} 5 \% 8 / \mathrm{y}$ & $1.162,98 \mathrm{f}$ & $4.070,43$ & 845,00 & 247,58 & 35,00 & $1.127,58$ & $2.942,85$ & 110 \\
\hline & $1 / 2 \mathrm{~S} \mathrm{~d} / 7 . \mathrm{ET} 2,5 \% 8 / \mathrm{y}$ & $1.104,77 \mathrm{~g}$ & $3.866,68$ & 704,17 & 129,31 & 35,00 & 868,47 & $2.998,21$ & 112 \\
\hline & $1 / 2 \mathrm{~S} \mathrm{~d} / 7 . \mathrm{ET} 5 \% 8 / \mathrm{y}$ & $1.106,91 \mathrm{~g}$ & $3.874,17$ & 704,17 & 247,58 & 35,00 & 986,74 & $2.887,43$ & 107 \\
\hline \multirow[t]{7}{*}{ PB 217} & $1 / 2 \mathrm{~S} \mathrm{~d} / 2$ (testemunha) & $1.508,13 b$ & $5.278,46$ & $2.112,50$ & 11,03 & 35,00 & $2.158,53$ & $3.119,92$ & 100 \\
\hline & $1 / 2 \mathrm{~S} \mathrm{~d} / 3$.ET $2,5 \% 8 / \mathrm{y}$ & $1.722,35 \mathrm{a}$ & $6.028,24$ & $1.408,33$ & 129,31 & 35,00 & $1.572,64$ & $4.455,60$ & 143 \\
\hline & $1 / 2 \mathrm{~S} \mathrm{~d} / 3$.ET $5 \% 8 / \mathrm{y}$ & $1.748,57 \mathrm{a}$ & $6.119,98$ & $1.408,33$ & 247,58 & 35,00 & $1.690,91$ & $4.429,07$ & 142 \\
\hline & $1 / 2 \mathrm{~S} \mathrm{~d} / 4$.ET $2,5 \% 8 / \mathrm{y}$ & $1.398,81 \mathrm{c}$ & $4.895,84$ & $1.056,25$ & 129,31 & 35,00 & $1.220,56$ & $3.675,29$ & 118 \\
\hline & $1 / 2 \mathrm{~S} \mathrm{~d} / 4 . \mathrm{ET} 5 \% 8 / \mathrm{y}$ & $1.385,98 \mathrm{c}$ & $4.850,93$ & $1.056,25$ & 247,58 & 35,00 & $1.338,83$ & $3.512,10$ & 113 \\
\hline & $1 / 2 \mathrm{~S} \mathrm{~d} / 5$.ET $2,5 \% 8 / \mathrm{y}$ & $1.206,38 \mathrm{e}$ & $4.222,32$ & 845,00 & 129,31 & 35,00 & $1.009,31$ & $3.213,02$ & 103 \\
\hline & $1 / 2 \mathrm{~S} \mathrm{~d} / 5 . \mathrm{ET} 5 \% 8 / \mathrm{y}$ & $1.314,47 \mathrm{~d}$ & $4.600,64$ & 845,00 & 247,58 & 35,00 & $1.127,58$ & $3.473,06$ & 111 \\
\hline
\end{tabular}

${ }^{(1)}$ Médias seguidas de letras iguais, para cada clone, não diferem entre si pelo teste de Tukey, a $1 \%$ de probabilidade. ${ }^{(2)} \mathrm{O}$ produto foi comercializado em forma de borracha seca, em março de 2006, por $\mathrm{R} \$ 3,50$. ${ }^{(3)} \mathrm{O}$ salário base foi $\mathrm{R} \$ 650,00$, incluídos $30 \%$ de encargos sociais por seringueiro por mês, considerando-se que um seringueiro, no sistema d/2, é responsável por 1.600 árvores; em d/3 por 2.400 árvores; em d/4 por 3.200 árvores; em d/5 por 4.000 árvores e em d/7 por 5.600 árvores. ${ }^{(4)}$ Verba destinada à compra de ácido acético e Ethrel, de acordo com a necessidade de cada sistema de sangria. ${ }^{(5)}$ Verba destinada à reposição de tigelas, bicas, arame, faca-de-sangria e esmeril, no caso de dano. ${ }^{(6)}$ Porcentagem de rentabilidade em relação à testemunha $(1 / 2 \mathrm{~S} \mathrm{~d} / 2)$, para cada clone. 


\section{Conclusões}

1. Em relação à média de produtividade, nos cinco anos de avaliação, os clones respondem bem ao sistema $1 / 2 \mathrm{~S} \mathrm{~d} / 3$ com estimulação com ethefon 2,5 e $5 \%$, com exceção do PB 235, que não responde ao estimulante.

2. Há baixa incidência de seca-do-painel no sistema $1 / 2 \mathrm{~S}$ d/7.ET 5\% 8/y, nos clones PB 260, PB 330 e PB 217; o PB 235 é suscetível ao distúrbio.

3. Os sistemas de sangria $1 / 2 \mathrm{~S}$ d/5.ET $2,5 \% 8 / \mathrm{y}$ ou $1 / 2 \mathrm{~S} \mathrm{~d} / 7$.ET $5 \% 8 / \mathrm{y}$, para o clone PB 260; $1 / 2 \mathrm{~S} \mathrm{~d} / 7$.ET 2,5\% 8/y, para o clone PB 235; e $1 \frac{1}{2} \mathrm{~S}$ d/3. ET $2,5 \% 8 / \mathrm{y}$ ou $1 \frac{1}{2} \mathrm{~S}$ d/3.ET $5 \%$ 8/y, para os clones PB 330 e PB 217, são superiores pelas vantagens econômicas e fisiológicas.

4. A rentabilidade com a expressiva redução de mão-deobra na frequiência d/7, em situações em que ocorra aumento no custo de mão-de-obra ou possível diminuição dos preços da borracha, permite ao produtor a redução na produção de borracha seca por hectare, com maior rentabilidade nos sistemas com menor frequiência de sangria, comparados aos sistemas com alta freqüência de sangria.

\section{Agradecimentos}

À Fapesp, pela concessão de bolsa; aos funcionários da Fazenda Santa Gilda, do grupo Rodobens Agrícola e Pecuária Ltda., pela colaboração no desenvolvimento do trabalho.

\section{Referências}

BERNARDES, M.S.; CASTRO, P.R. de C. e; FURTADO, E.L.; SILVEIRA, A.P. da; COSTA, J.D.; MARTINS, A.N.; VIRGENS FILHO, A.C. Manual de sangria da seringueira. São José do Rio Preto: Bridgestone/Firestone do Brasil, 1995. 20p.

BERNARDES, M.S.; CASTRO, P.R. de C. e; MARTINS, A.N.; VIRGENS FILHO, A. de C. Fatores ligados à escolha do sistema de explotação. In: BERNARDES, M.S. (Ed.). Sangria da seringueira. Piracicaba: Esalq/USP, 2000. p.139-182.

CONDURU NETO, J.M.H. Sistema de explotação com frequiência reduzida de sangria e uso de estimulantes. In: ENCONTRO NACIONAL SOBRE EXPLOTAÇÃO E ORGANIZAÇÃO DE SERINGAIS DE CULTIVO, 1., 1986, Brasília. Anais. Brasília: Sudhevea, 1986. p.45-58.

EMBRAPA. Centro Nacional de Pesquisa de Seringueira e Dendê(Manaus, AM). Melhoramento genético da seringueira. Manaus: EmbrapaCNPSD, 1987. 23p. (Embrapa-CNPSD. Documentos, 10).

GONÇALVES, P. de S.; BATAGLIA, O.C.; ORTOLANI, A.A.; FONSECA, F. da S. Manual de heveicultura para o Estado de São Paulo. Campinas: Instituto Agronômico, 2001. 78p.
GONÇALVES, P. de S.; CARDOSO, M.; ORTOLANI, A.A. Origem, variabilidade e domesticação da Hevea: uma revisão. Pesquisa Agropecuária Brasileira, v.25, p.135-156, 1990.

GONÇALVES, P. de S.; SILVA, M. deA.; GOUVÊA, L.R.L.; SCALOPPI JUNIOR, E.J. Genetic variability for girth growth and rubber yield characters in Hevea brasiliensis. Scientia Agricola, v.63, p.246-254, 2006.

GONÇALVES, P. de S.; SOUZA, S.R. de; BRIOSCHI, A.P.; VIRGENS FILHO, A. de C.; MAY, A.; CAPELA-ALARCON, R.S. Efeito da frequiência de sangria e estimulação no desempenho produtivo e econômico de clones de seringueira. Pesquisa Agropecuária Brasileira, v.35, p.10811091, 2000.

IBGE. Produção da extração vegetal e da silvicultura. Rio de Janeiro: IBGE, 2004. 59p. v.19. Disponível em: <http://www.ibge.gov.br/home/ estatistica/economia/pevs/2004/.pdf>. Acesso em: 17 mai. 2007.

IRSG - INTERNATIONAL RUBBER STUDY GROUP. Rubber Statistical Bulletin. Wembley: IRSG, 2007. v.61. 61p.

PINHEIRO, E.; CONCEIÇÃO, H.E.O.; PINHEIRO, F.S.V.; VIÉGAS, J.M.; ARANTES, M.A.L. A reabilitação da seringueira na Amazônia. In: CICLO DE PALESTRAS SOBRE A HEVEICULTURA PAULISTA, 3., 2002, São José do Rio Preto. Anais. São José do Rio Preto: SAA, Apabor, 2002. p.38-62.

PRADO, H. do. Solos do Brasil: gênese, morfologia, classificação, levantamento, manejo agrícola e geotécnico. 3.ed. Piracicaba: H. do Prado, 2003. 275p.

PREMAKUMARI, D.; PANIKKAR, A.O.N.; SETHURAJ, M.R.; MARATTUKALAM, J.G. Associations of structural traits: yield, girth and occurrence of tapping panel dryness in Hevea brasiliensis. Indian Journal of Natural Rubber Research, v.10, p.27-33, 1997.

RAJAGOPAL, R.; VIJAYAKUMAR, K.R.; THOMAS K.U.; KARUNAICHAMY, K. Effect of judicious ethephon application on yield response of Hevea brasiliensis (Clone RRII 105) under $1 / 2 \mathrm{~S} \mathrm{~d} / 36 \mathrm{~d} / 7$ tapping system. Journal of Rubber Research, v.7, p.138-147, 2004.

RRIM - RUBBER RESEARCH INSTITUTE OF MALAYSIA. RRIM planting recommendations 1989-91. Planters' Bulletin of the Rubber Research Institute of Malaysia, v.198, p.3-23, 1989.

SAA. Melhoramento genético da Seringueira (Hevea spp.). Projeto Temático - Fapesp. Campinas, 2006. 219p. Relatório Técnico.

STEEL, R.G.D.; TORRIE, J.H. Principles and procedures of statistics. New York: McGrow-Hill, 1980. 631p.

TOLEDO, P.E.N. de; GHILARDI, A.A. Custo de produção e rentabilidade do cultivo da seringueira no Estado de São Paulo. Informações Econômicas, v.30, p.30-43, 2000.

USHA NAIR, N.; RAMESH NAIR, B.; THOMAS, M.; GOPALAKRISHNAN, J. Latex diagnosis in relation to exploitation systems in clone RRII 105. Journal of Rubber Research, v.7, p.127137, 2004.

VIRGENS FILHO, A.C.; SENA GOMES,A.R.; SANTOS, S.M. Sistemas de sangria em painel baixo e painel alto do clone Fx 25. In: ENCONTRO NACIONAL SOBRE EXPLOTAÇÃO E ORGANIZAÇÃO DE SERINGAIS DECULTIVO, 1.,1986, Brasília. Anais. Brasília: Sudhevea, 1986. p.35-44. 